\title{
PARP1-siRNA suppresses human prostate cancer cell growth and progression
}

\author{
YONGCHANG LAI $^{1 *}$, ZHENZHEN KONG $^{1 *}$, TAO ZENG $^{1 *}$, SHAOHONG XU ${ }^{1,2^{*}}$, XIAOLU DUAN $^{1}$, \\ SHUJUE LI ${ }^{1}, \mathrm{CHAO} \mathrm{CAI}^{1}$, ZHIJIAN ZHAO ${ }^{1}$ and WENQI WU ${ }^{1}$ \\ ${ }^{1}$ Department of Urology, Minimally Invasive Surgery Center, The First Affiliated Hospital of Guangzhou Medical University, \\ Guangzhou Urology Research Institute, Guangdong Key Laboratory of Urology, Guangzhou 510230; \\ ${ }^{2}$ Department of Urology, Shangrao People's Hospital, Shangrao, Jiangxi 334000, P.R. China
}

Received September 27, 2017; Accepted January 25, 2018

DOI: $10.3892 /$ or.2018.6238

\begin{abstract}
Poly (ADP-ribose) polymerase (PARP) inhibitors, such as olaparib or rucaparib, have shown treatment efficacy in BRCA1/2-deficient tumors. However, since PARP inhibitors (PARPi) mainly modulate the activation of PARP but not its expression, whether small interfering RNA (siRNA) specific to PARP has the same function as PARPi has not been well defined. In the present study it was demonstrated that PARP1-siRNA could reduce prostate cancer (PCa) cell progression regardless of the BRCA1/2 mutation. PARP1 silencing could significantly inhibit PC3 cell migration and invasion. Additionally, PARP1siRNA also inhibited the proliferation of PC3 and Du145 cells. After the induction of apoptosis by docetaxel, cleavedcaspase 3 of DU145 and C4-2 cells increased significantly in the PARP1-siRNA group. In the xenograft nude mouse model, PARP1-siRNA could suppress xenograft tumor size of PC3 cells and produce a more regular morphology. In vitro and in vivo, PARP1 silencing significantly downregulated vimentin expression and upregulated E-cadherin expression, both of which are epithelial-mesenchymal transition (EMT) markers. It has been revealed that PI3K inhibition could sensitize the effect of PARPi. Notably, PARP1-siRNA could suppress the expression of EGFR and p-GSK3 $\beta$ (Ser9) in PCa cells, which was different from PARPi. Our results indicated that PARP1-siRNA can suppress the growth and invasion capacity of PCa cells, thereby suggesting that PARP1-siRNA, which is different from PARPi, may provide a potential treatment method for PCa.
\end{abstract}

Correspondence to: Professor Wenqi Wu, Department of Urology, Minimally Invasive Surgery Center, The First Affiliated Hospital of Guangzhou Medical University, Guangzhou Urology Research Institute, Guangdong Key Laboratory of Urology, Kangda Road 1, Haizhu, Guangzhou, Guangdong 510230, P.R. China

E-mail:wwqwml@163.com

*Contributed equally

Key words: PARP1, PC3 cells, prostate cancer, Du145 cells, siRNA, EGFR

\section{Introduction}

Prostate cancer ( $\mathrm{PCa}$ ) is the most common malignant tumor of the male genital system. It was estimated in 2017, that 161,360 new cancer cases were diagnosed and 26,730 deaths occurred in the US (1). Androgen receptor signaling is crucial for the initiation and progression of prostate cancer (2). Initially, most PCa cells respond to castration therapy, but eventually tumors relapse and take on a castration therapy-resistant prostate cancer (CRPC) phenotype that correlates with poor prognosis and high metastatic potential. Although targeted drugs and immune checkpoint blockade drugs have rapidly developed recently, mCRPC has exhibited resistance to these treatments (3), thus novel drug combination regimens or therapeutic approaches to combat PCa are still urgently needed.

Poly (ADP-ribose) polymerases (PARPs) are a family of related enzymes, and PARP1 is the founding and best characterized member (4). PARP1 is activated after binding to DNA strand breaks and uses $\mathrm{NAD}^{+}$as a substrate to form long branched polymers of poly (ADP-ribose) (PAR). PARP1 is involved in many cellular processes, including various DNA repair pathways and in the maintenance of genomic stability (5). As a ubiquitously expressed $\mathrm{NAD}^{+}$-dependent nuclear enzyme, PARP1 has prognostic value for many human cancers (6). Homologous recombination (HR) defects renders PCa sensitive to PARP inhibition (7). However, the use of PARP inhibitors (PARPi) has been limited to repair-deficient cancers (8).

PARPi suppresses PARP activation but not its expression. However, whether the function of small interfering RNA (siRNA) specific to PARP is identical to PARPi has not been well defined. Our previous study revealed that PARP1 and PAR expression were markedly elevated in PCa tissues compared to benign prostate hyperplasia (BPH) tissues (9), and that inhibition of PARP1 by siRNA could enhance the activity of docetaxel against PC3 cells (10). However, the exact effect of PARP1-siRNA in PCa cell growth and progression and its mechanism remain unclear. In the present study, we investigated whether inhibition of PARP1 by siRNA could reduce the growth and invasion capacity of $\mathrm{PCa}$ cell lines regardless of the BRCA1/2 mutation. 


\section{Materials and methods}

Cell culture and drugs. Du145, C4-2 and PC3 cells were purchased from the American Type Culture Collection (ATCC; Manassas, VA, USA). Du145, C4-2 and PC 3 cells were cultured in Dulbecco's modified Eagle's medium (DMEM), RPMI-1640 and DMEM/F12 medium respectively, with 10\% (v/v) fetal bovine serum (FBS; Gibco/Life Technologies; Thermo Fisher Scientific, Inc., Grand Island, NY, USA) and $1 \%(\mathrm{v} / \mathrm{v})$ penicillin and streptomycin at $37^{\circ} \mathrm{C}$ in a humidified incubator supplemented with $5 \% \mathrm{CO}_{2}$. Docetaxel was obtained from Selleck Chemicals (Houston, TX, USA). PC3 monoclonal cells stably expressing pGPU6-GFP-Neo-PARP1-siRNA-1706 or pGPU6-GFP-Neo-PARP1-siRNA-NC were generated and cultured in the presence of $\mathrm{G} 418(0.5 \mathrm{mg} / \mathrm{ml})$.

RNA interference and transfection. siRNA that targeted the human PARP1 gene was synthesized by RiboBio Co., Ltd. (Guangzhou, China). The synthesized siRNA sequences were the following: siRNA-NC, 5'-UUCUCCGAACGUGUCACG UTT-3'; siRNA-1706,5'-GAGGAAGGUAUCAACAAAUTT-3'; siRNA-2003, 5'-GAGCACUUCAUGAAAUUAUTT-3'; and siRNA-2907, 5'-GAGACCCAAUAGGUUAAUTT-3'.

Transfection of synthesized siRNAs was carried out using Lipofectamine 2000 (Invitrogen; Thermo Fisher Scientific, Inc., Carlsbad, CA, USA) according to the manufacturer's instructions.

Cell viability assessment. Du145 cells were plated in 96-well plates. After $24 \mathrm{~h}$, the cells were transfected with PARP1-siRNA-NC or PARP1-siRNA-1 using a riboFECT ${ }^{\text {TM }}$ CP Transfection Kit from RiboBio Co., Ltd. (Guangzhou, China). Approximately $72 \mathrm{~h}$ later, the cells were replaced with DMEM without FBS and incubated for another $24 \mathrm{~h}$. 3-(4,5-Dimethylthiazol-2-yl)-2,5-diphenyltetrazolium bromide (MTT) was added to cells for a 3 to 4-h reaction, and dimethyl sulfoxide (DMSO) was applied to dissolve the sediment. The optical density of the MTT results was assessed at a wavelength of $490 \mathrm{~nm}$ and the cell survival rate was normalized.

In vitro migration assay. Cells were transfected with the indicated siRNA and cultured to confluence. Then, an artificial scratch wound was generated using a $200-\mu 1$ pipette tip, and cells were washed to remove the detached cells and debris. Cell migration was captured using an inverted microscope and evaluated by measuring the difference in the wound width and counting the migrated number of cells.

In vitro colony formation assay. Forty-eight hours after transfection, cells were washed with phosphate-buffered saline (PBS), trypsinized, and reseeded into 6-pore dishes at a density of 500 cells/well. Under the same culture conditions, the cells were cultured for another 9-12 days, and then fixed and dyed with $4 \%$ crystal violet in ethanol. The number of cell colonies was calculated.

In vitro invasion assay. Cell invasion was evaluated using a Transwell ${ }^{\circledast}$ permeable support invasion chamber (Corning Incorporated, Corning, NY, USA) according to the manufacturer's protocol. Forty-eight hours after transfection, the cells were cultured in the insert for $48 \mathrm{~h}$ and stained with Mayer's hematoxylin. Eight fields in each sample were imaged, and invasion was evaluated by counting the invaded number of cells.

In vivo xenograft model. All animal experimental procedures were carried out in accordance with a mouse protocol, which was approved by the Animal Research Committee of Guangzhou Medical University. Male nude mice (aged 8 weeks; weighing $18-20 \mathrm{~g}$ ) were fed a standard diet and water during the study. Fifteen mice were randomly assigned to 3 experimental groups evenly (5 mice in each group): PC3-siRNA-1706, PC3-siRNA-NC and PC3 C (control). PC3 monoclonal cells stably expressing pGPU6-GFP-NeoPARP1-siRNA-1706 or pGPU6-GFP-Neo-PARP1-siRNA-NC were inoculated subcutaneously in the flank regions of the mice to establish the tumor model. Following subcutaneous inoculation, the mice were examined daily for tumor formation and growth. The sizes of the subcutaneous tumors were monitored twice weekly by assessing the length and width. The tumor volumes were calculated using the formula: Volume $\left(\mathrm{mm}^{3}\right)=$ length $(\mathrm{mm}) \mathrm{x}$ width ${ }^{2}\left(\mathrm{~mm}^{2}\right) / 2$. At 40 days after inoculation, all experimental mice were euthanized for further study.

Western blot analysis. For protein extraction, cells or homogenized tissue were rinsed with PBS and treated in ice-cold lysis buffer. After scrape, collection and ultra-sonication, the lysate solutions were centrifuged at 12,000 RCF for $30 \mathrm{~min}$ at $4^{\circ} \mathrm{C}$. The Bradford protein assay method was used to assess the protein concentration. Equal amounts of protein were separated on a $10 \%$ SDS polyacrylamide gel and transferred to a NC membrane (Millipore, Billerica, MA, USA). The membranes were blocked in 5\% non-fat dry milk in PBS-T for $2 \mathrm{~h}$ at room temperature and incubated with primary antibodies appropriately diluted in PBS-T overnight at $4^{\circ} \mathrm{C}$. Primary antibodies against PARP1 (1:200; mouse, monoclonal; cat. no. sc-8007), vimentin (1:200; mouse, monoclonal; cat. no. sc-6260) and GAPDH (1:1,000; mouse, monoclonal; cat. no. sc-47724) were obtained from Santa Cruz Biotechnology (Santa Cruz, CA, USA). EGFR (1:2,000; rabbit, monoclonal; cat. no. 1902-1) was obtained from Epitomics (Cambridge, UK). Anti-tubulin (1:2,000; rabbit, ployclonal; cat. no. 2148), GSK3- $\beta(1: 1,000$; rabbit, monoclonal; cat. no. 12456), pGSK3- $\beta$ (ser9) $(1: 1,000$; rabbit, monoclonal; cat. no. 5558), E-cadherin (1:1,000; mouse, monoclonal; cat. no. 14472), Cx43 (1:1,000; rabbit, ployclonal; cat. no. 3512) and cleaved-caspase 3 (1:1,000; rabbit, monoclonal; cat. no. 9664) were purchased from Cell Signaling Technology, Inc. (Danvers, MA, USA). The membranes were incubated with secondary antibodies appropriately diluted in PBS-T for $2 \mathrm{~h}$ at room temperature. The secondary antibodies were anti-mouse or anti-rabbit IgG conjugated to HRP (Santa Cruz Biotechnology). The membranes were developed for chemiluminescence detection using an ECL detection kit (Thermo Fisher Scientific, Inc., Waltham, MA, USA). The band intensities were quantified using ImageJ software, and all western blot analyses were repeated 3 times.

Quantitative real-time PCR analysis. Total RNA was extracted with a MiniBEST Universal RNA Extraction kit 
(Takara, Otsu, Japan). An equal amount of RNA was reverse transcribed to cDNA and amplified by PCR according to the manufacturer's protocol (Takara). Quantitative real-time PCR was performed using the SYBR-Green PCR Master mix (Takara) according to the manufacturer's protocol. The primers were as follows: PARP1 forward, 5'-AAGGCGAAT GCCAGCGTTAC-3' and reverse, 5'-GGCACTCTTGGAGAC CATGTCA-3'; E-cadherin forward, 5'-TCCTCCCAATAC ATCTCCCTTCA-3' and reverse, 5'-TCTCCGCCTCCTTCT TCATCATA-3'; vimentin forward 5'-TTCGCCAACTACATC GACAAGG-3'and reverse, 5'-TTCAAGGTCAAGACGTGC CAG-3'; GAPDH forward, 5'-GCACCGTCAAGGCTGAGA AC-3'and reverse, 5'-AUAAUUUCAUGAAGUGC-UCTT-3'. The real-time PCR reaction was maintained at $95^{\circ} \mathrm{C}$ for $5 \mathrm{~min}$, followed by 40 cycles at $95^{\circ} \mathrm{C}$ for $15 \mathrm{sec}, 57^{\circ} \mathrm{C}$ for $30 \mathrm{sec}$ and $72^{\circ} \mathrm{C}$ for $20 \mathrm{sec}$. All samples were processed in triplicate, and all values were normalized to the expression levels of GAPDH. The relative quantification was determined by the comparative Ct method.

Hematoxylin and eosin $(H \& E)$ staining. The tumor tissues were washed with PBS, fixed with $4 \%$ paraformaldehyde, paraffin-embedded, and sliced to a $3-\mu \mathrm{m}$ thickness to generate serial sections. Slices were dewaxed in xylene for $10 \mathrm{~min}$ and placed into a mixture of alcohol and xylene (1:1) for $\sim 5 \mathrm{~min}$. After the slices were sequentially immersed in 100, 95, 85 and $70 \%$ alcohol for 2 min per step, they were placed in distilled water before staining. Then, the slices were stained with hematoxylin for $\sim 10 \mathrm{~min}$. Subsequently, the slices were immersed into water for $1 \mathrm{sec}$, and then $0.5-1 \%$ hydrochloric acid alcohol was used for a short time to clear the excess hematoxylin. The time was controlled by microscopy until the nuclei and nuclear chromatin were clear. After rinsing with water for 15-30 min, the nuclei became blue, and the slices were rinsed again in distilled water (short time). Thereafter, $0.5 \%$ eosin was applied to stain the slices for $1 \mathrm{~min}$, and the slices were sequentially immersed in $70,85,95$, and $100 \%$ alcohol for dehydration for 2-3 min per step. The slices were then cleared with xylene two times (5 min/time). Excess xylene in the sections was wiped away, and the specimens were not dried. A small amount of neutral gum was quickly added, and the glass was covered to seal the specimens.

Immunohistochemistry. All sections were formalin-fixed, paraffin-embedded and stained according to the standard immunohistochemistry protocol. Following incubation with primary antibodies for PARP1, E-cadherin and vimentin, the sections were incubated with biotinylated secondary antibodies (Maixin Biotech Co., Ltd., Fuzhou, China). Subsequently, the avidin-biotin horseradish peroxidase complex and diaminobenzidine chromogen (Maixin Biotech Co., Ltd.) were used as the detection system. Each stained slide was assessed by an experienced laboratory technician independently in a blinded fashion without any information regarding the group.

Statistical analysis. Statistical analysis was performed using SPSS 16.0 software (SPSS, Inc., Chicago, IL, USA). All data are expressed as the mean \pm SD. The mean differences $(\mathrm{P}<0.05)$ were compared using a one-way ANOVA ( $>2$ groups $)$ or Student's t-test (2 groups). Western blot data was analyzed with Image J software. In the figures, an asterisk represents $\mathrm{P}<0.05$ compared to the corresponding group and indicates a statistically significant result.

\section{Results}

siRNA-mediated PARPl inhibition suppresses migration, invasion and proliferation of PC3 cells in vitro. Two small interfering RNAs (1706 and 2003) used to knock down the expression of PARP1 in PC3 cells in our previous study (10), were synthesized and transfected into PC3 cells. Real-time PCR and western blot analyses revealed that both PARP1 siRNAs, 1706 and 2003, significantly decreased PARP1 mRNA and protein expression compared to the negative control in PC3 cells (Fig. 1A and B). Then, we investigated the effect of PARP1-siRNA on the migration and invasion capacity of PC3 cells. The results of the wound healing assays revealed that both siRNA-1706 and siRNA-2003 significantly suppressed the migration capacities of PC3 cells in vitro (Fig. 1C). The results of the Transwell invasion assays revealed that both siRNA-1706 and siRNA-2003 significantly suppressed the invasion capacities of PC3 cells in vitro (Fig. 1D).

Since siRNA-1706 was more effective than siRNA-2003 in suppressing the expression of PARP1, siRNA-1706 was further used in the following experiment. Colony formation assays were analyzed, and the results revealed that the siRNA-1706 group grew significantly more slowly than the negative control group (Fig. 1E). In addition, our previous study confirmed that PARP1 siRNAs could inhibit the EGFR/Akt/FoxO pathway. In the present study, we found that PARP1-siRNA could inhibit p-GSK-3 $\beta$ which is also downstream of AKT (Fig. 1F). Therefore, PARP1 knockdown may suppress PCa progression by inhibiting EGFR/Akt and its downstream including the FoxO and GSK-3 $\beta$ signaling pathway. To further investigate whether PARP1 knockdown reduced the tumorigenesis and metastasis capacity of PCa cells in vivo, we constructed a stable PARP1 knockout model in PC3 cells by recombinant plasmid. The PCR and western blot results revealed that stable transfection of the plasmid could effectively inhibit the expression of PARP1 (Fig. 1G).

siRNA-mediated PARPI inhibition reduces the growth of PC3 cells in vivo. After a stable PARP1 knockout model was constructed and confirmed, we investigated whether PARP1 knockdown could reduce the tumor growth and tumorigenesis capacity of PCa cells in vivo. The results revealed that in comparison with the PC3 control (C) group and the PC3-siRNA-negative control group (NC), the tumor growth in the PC3-siRNA-1706 group was markedly reduced (Fig. 2A and B). The mean volumes and wet weights of the tumors from the PC3-siRNA-1706 group were significantly decreased in comparison with the PC3 control group and the PC3-siRNA-NC group (Fig. 2C and D). The H\&E staining results revealed that the control group and no-load group promoted a larger cell volume, rich in cytoplasm, in which the nucleus was larger and deeply stained; the nuclei were round and had prominent nucleoli. In this group, mitotic activity was more common, the nucleus/cytoplasm ratio was increased, cells were rich in cytoplasm, and there was a higher number of interstitial vessels. The PC3-siRNA-1706 group 
A mRNA expression level of PC 3 cells

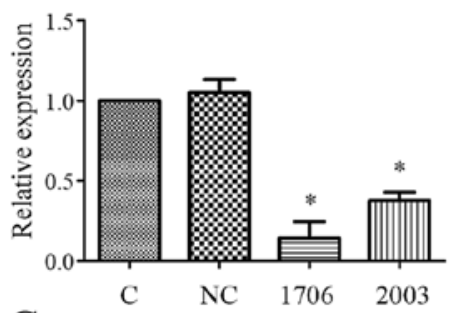

$\mathrm{C}$

Wound healing experiments in PC3 cells

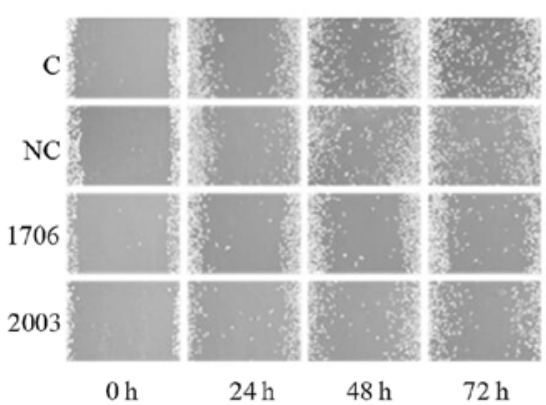

E

Colony formation assay of PC 3 cells

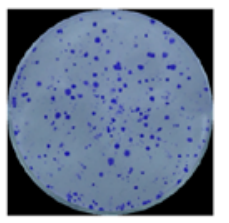

$\mathrm{NC}$

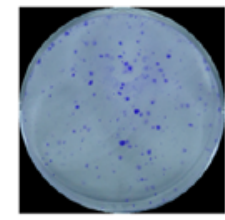

1706
$\mathrm{B}$

Protein expression level of PC3 cells
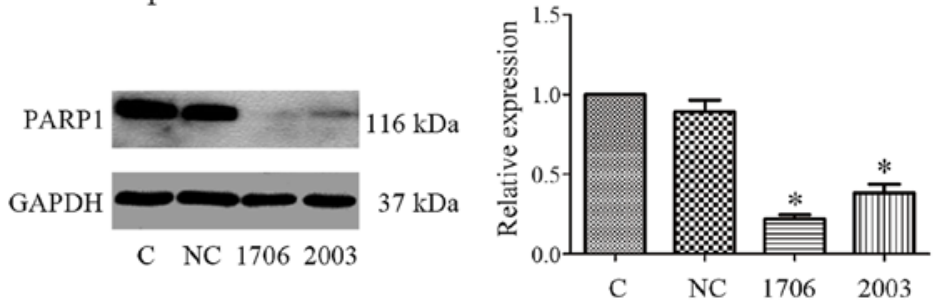

D Invasion assays of PC 3 cells

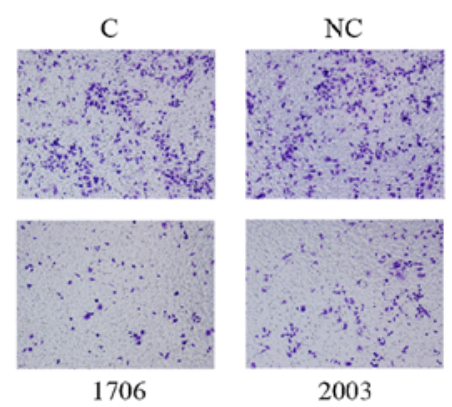

$\mathrm{F}$

PARP1 and $\mathrm{p}-\mathrm{GSK} 3-\beta$ in PC3 cells
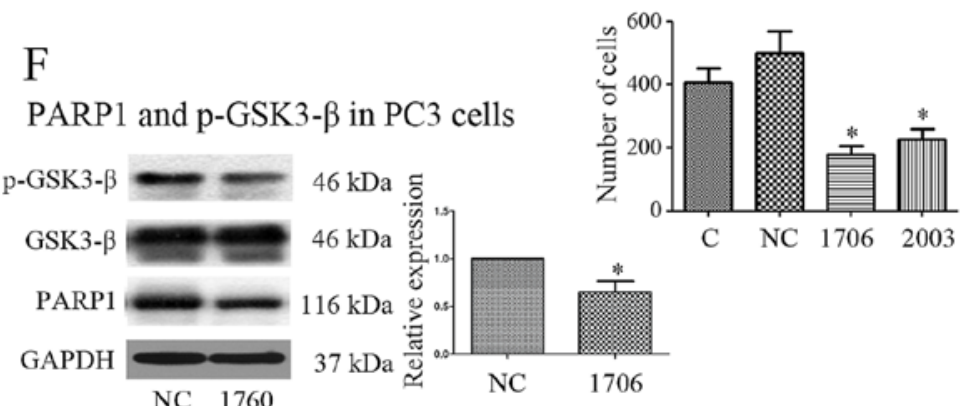

C NC $1706 \quad 2003$

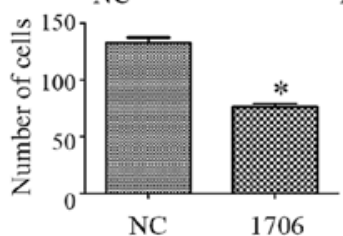

G Stable PARP1 knockout PC3 cell model for mice xenograft tumor experiment
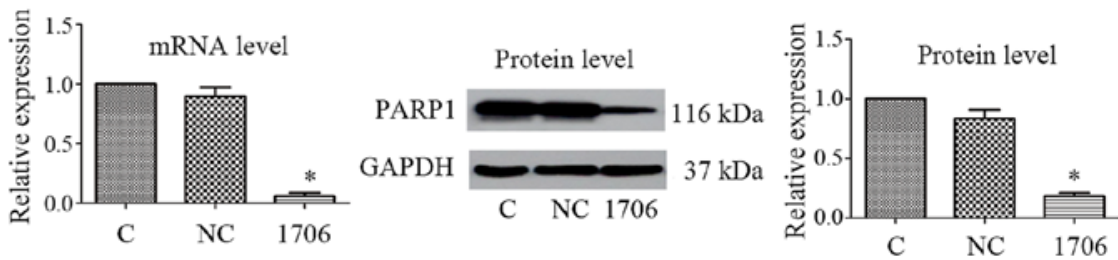

Figure 1. siRNA-mediated PARP1 inhibition suppresses the migration, invasion and growth capacities of PC3 cells in vitro. (A and B) Following transfection with the indicated siRNA, the expression of PARP1 and GAPDH in PC3 cells was detected by real-time PCR and western blotting. Following transfection with the indicated siRNA in PC3 cells, (C) cell migration capacity was detected by wound healing assay, (D) cell invasion capacity was detected by Transwell invasion assay, (E) cell proliferation ability was assessed by colony formation assay, and (F) the expression of p-GSK-3 $\beta$ (Ser9) was detected by western blotting. (G) A stable model of transfection with the indicated siRNA in PC3 cells to perform xenograft tumor experiments was constructed, and the expression of PARP1 and GAPDH was detected by real-time PCR and western blotting. $\mathrm{n}=3$. " $\mathrm{P}<0.05$ with respect to the control group. $\mathrm{C}$, control group; NC, negative control group; 1706, siRNA-1706 group; 2003, siRNA-2003 group.

exhibited smaller PC3 cells in the nude mice with smaller cytoplasms, in which the nuclei were small, round and lighter in color; the nucleoli were obvious, the nucleus/cytoplasm ratio was smaller, and there were fewer interstitial blood vessels (Fig. 2E).

PARPI silencing and EMT molecular marker expression. Since EMT is a key step for cancer cells to acquire invasion and metastasis abilities (11), we analyzed the expression of some EMT-associated markers in PC3 cells by PARP1 knockdown. Real-time PCR analysis revealed that PARP1 knockdown significantly upregulated the mRNA level of E-cadherin, which was accompanied with significant downregulation of vimentin mRNA (Fig. 3A). The western blot analysis results further validated the increased E-cadherin expression and decreased vimentin expression in PC 3 cells after knockdown of PARP1 (Fig. 3B). To further explore whether PARP1 knockdown suppressed EMT in PCa cells in vivo, we detected the expression of EMT-associated markers in xenograft tumors. Consistent with the in vitro results, real-time PCR and western blot analyses revealed the same results in vivo (Fig. 3C and D). Immunohistochemical analysis 
A Xenograft tumors of nude mice

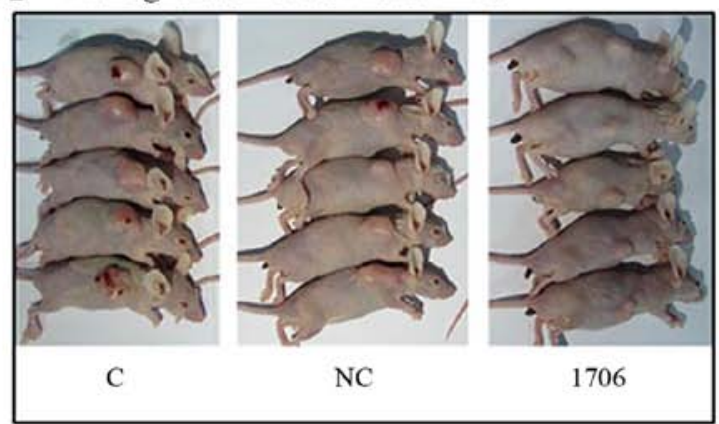

C Dissected prostate tumors in nude mice
B Growth curve of tumors in nude mice

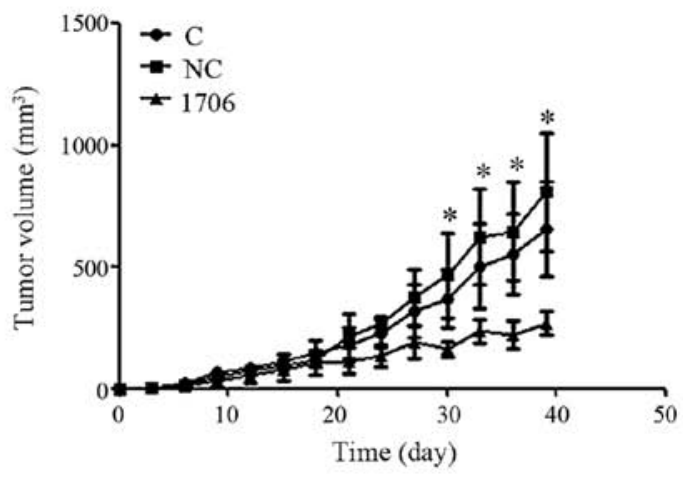

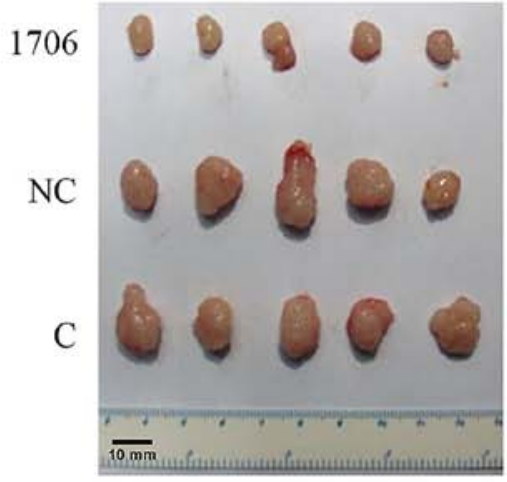

D Tumor volume and wet weight of nude mice
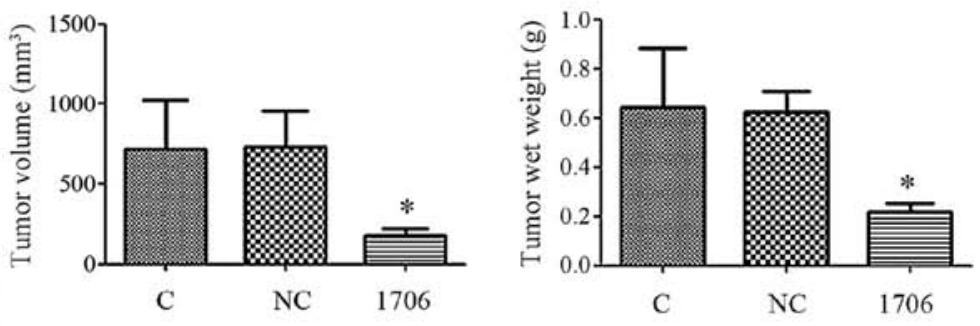

E H\&E staining of xenograft tumors from nude mice

$\mathrm{C}$

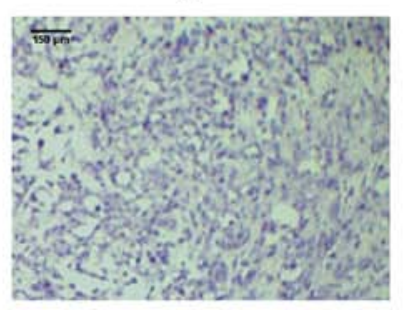

$\mathrm{x} 400$

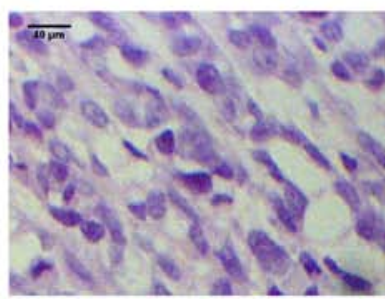

NC
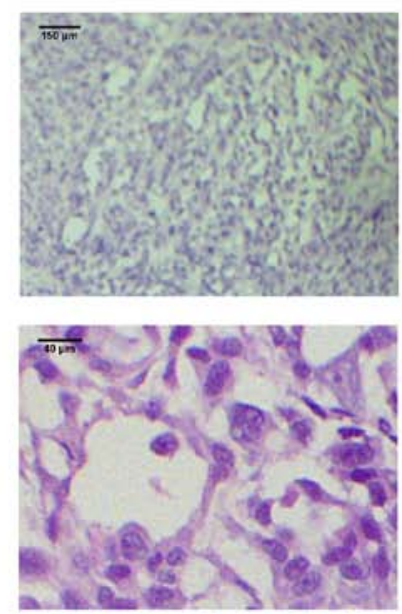

1706
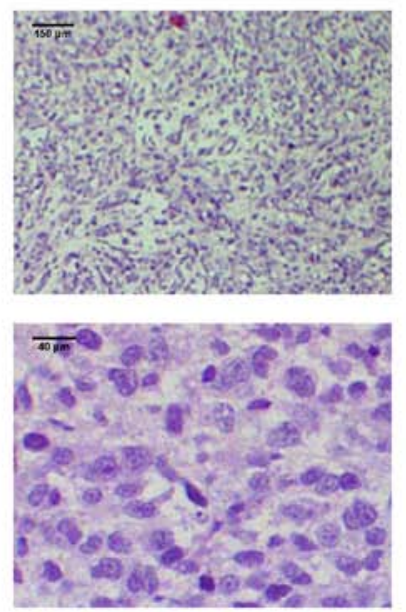

Figure 2. siRNA-mediated PARP1 inhibition suppresses the growth capacities of PC3 cells in vivo. (A) Images display representative nude mice bearing xenograft tumors formed by subcutaneous inoculation of PC 3 monoclonal cells. (B) Growth curve of the tumors formed by subcutaneous inoculation of PC 3 monoclonal cells in nude mice. (C) Images display the dissected prostate tumors formed by subcutaneous inoculation of PC 3 monoclonal cells in nude mice (with a scale of $10 \mathrm{~mm}$ ). (D) The volumes and wet weights of dissected prostate tumors were assessed. (E) H\&E staining of dissected prostate tumors from nude mice (x100 magnification with a scale of 150 microns and x400 magnification with a scale of $40 \mathrm{microns}$ ). $\mathrm{n}=5$. $\mathrm{P}<0.05$ with respect to the control group.

revealed that, compared with the $\mathrm{PC} 3$ control group and the PC3-siRNA-NC group, the PC3-siRNA-1706 group had a higher level of the E-cadherin protein and a lower level of the vimentin protein (Fig. 3E).

siRNA-mediated PARPl inhibition suppresses the EGFR signaling pathway in C4-2 and DU145 cells. In order to further confirm the function of PARP1 siRNAs in other PCa cells, siRNA-1706, siRNA-2003, siRNA-2907 and non-specific siRNA [negative control (NC)] were used to knock down the expression of PARP1 in C4-2 cells. The results revealed that siRNA-1706 was the most efficient and was further used in the following experiment (Fig. 4A). After PARP1 knockdown by PARP1 siRNA-1706, EGFR was decreased in C4-2 cells (Fig. 4B). After PARP1 was knocked down by PARP1 siRNA-1706 in C4-2 cells, docetaxel was used to induce apoptosis and the expression of cleaved-caspase 3 was detected via western blotting (Fig. 4C). Connexin 43 (Cx43) may be associated with apoptosis since it is a component of gap junctions, therefore its expression was also detected (Fig. 4C). 


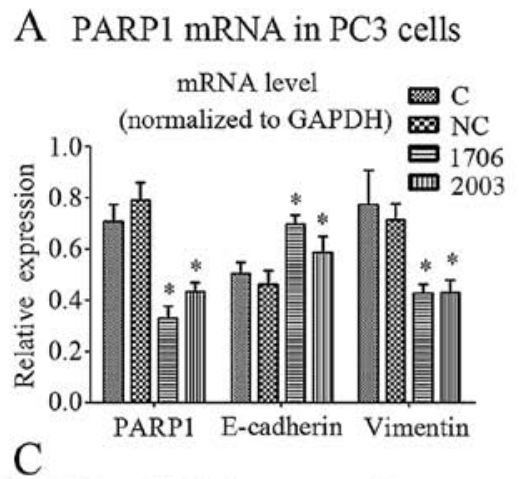

B PARP1 protein in PC3 cells

Protein level (normalized to GAPDH)

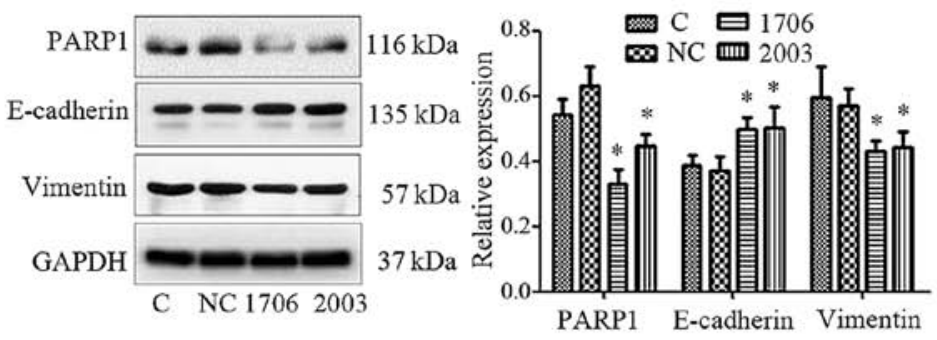

PARPI mRNA in xenograft tumors of mice

D

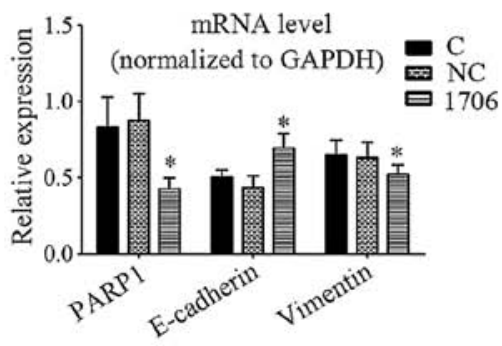

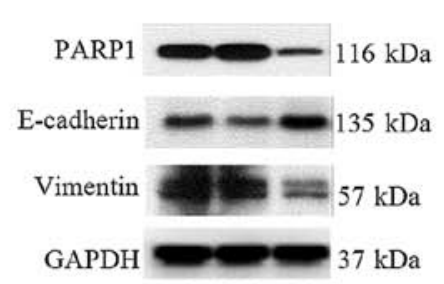

C NC 1706
E Immunohistochemical analysis of xenograft tumors
$\mathrm{C}$
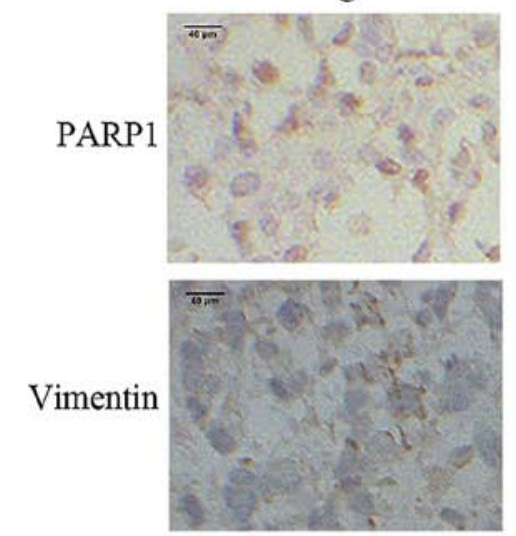

E-cadherin

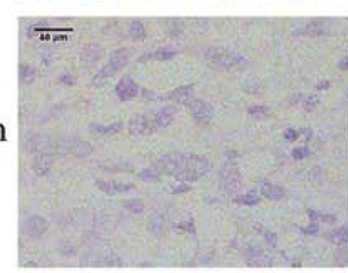

$\mathrm{NC}$
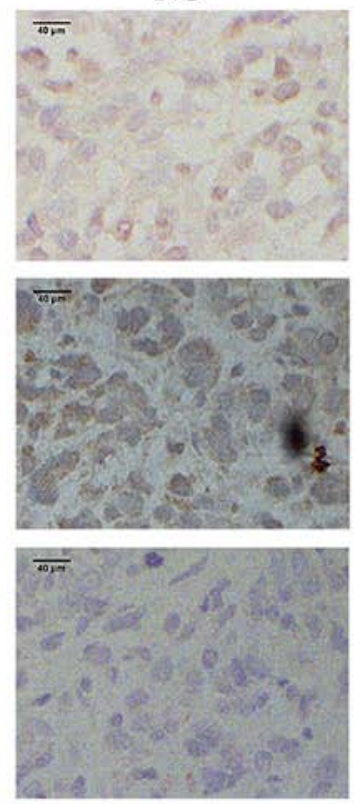

1706
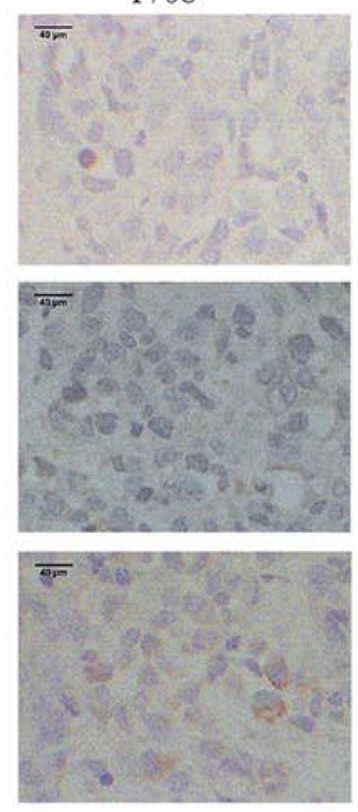

Figure 3. PARP1-siRNA and epithelial-mesenchymal transition markers of PC3 cells in vitro and in vivo. (A and B) siRNA-mediated PARP1 inhibition suppresses epithelial-mesenchymal transition in vitro. (C and D) siRNA-mediated PARP1 inhibition suppresses epithelial-mesenchymal transition in vivo. (E) Immunohistochemical analysis of siRNA-mediated PARP1 inhibition on epithelial-mesenchymal transition in vivo (x400 magnification with a scale of 40 microns). $n=3$. $P<0.05$ with respect to the control group.

Compared with the NC group, EGFR expression in the PARP1 siRNA-1706 group was significantly decreased in Du145 cells (Fig. 4D). Similar to the C4-2 cells, cleavedcaspase 3 and $\mathrm{Cx} 43$ expression in the siRNA-1706 group was significantly increased with docetaxel $(100 \mathrm{nM})$ treatment in Du145 cells (Fig. 4E). After PARP1 was knocked down by PARP1 siRNA-1706 in Du145 cells, its proliferation was decreased (Fig. 4F). Collectively, these results indicated that PARP1 knockdown reduced the progression and metastasis capacities of PCa cells both in vitro and in vivo.

\section{Discussion}

$\mathrm{PCa}$ is a major health problem in older men worldwide. Progression and metastasis are considered to be major challenges for PCa treatment. Therefore, studies on the mechanism of PCa progression and metastasis, and the corresponding therapeutic methods are urgently required. In the present study, our results indicated that PARP1-siRNA could suppress the progression and invasion capacity of $\mathrm{PCa}$ cells regardless of the BRCA1/2 mutation by inhibiting the EGFR/GSK-3 $\beta$ 
A PARP1 siRNA in C4-2 cells
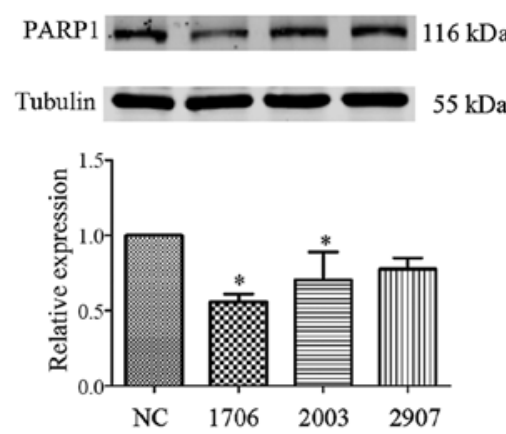

D PARP1 and EGFR in Du145 cells
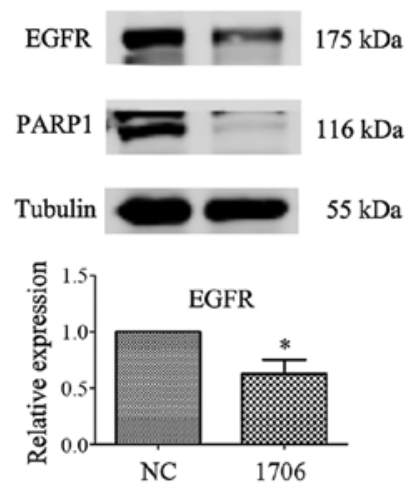

B PARP1 and EGFR in C4-2 cells
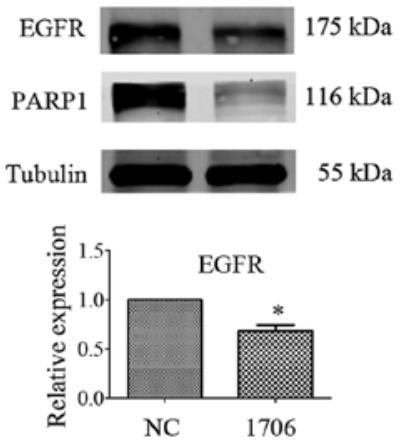

E Apoptosis in Du145 cells
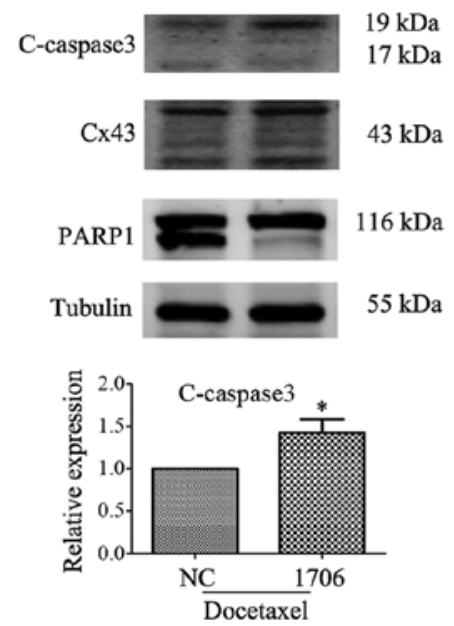

C Apoptosis in C4-2 cells
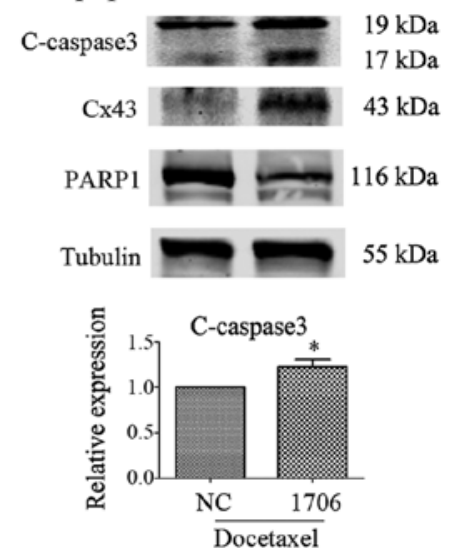

F Proliferation in Du145 cells

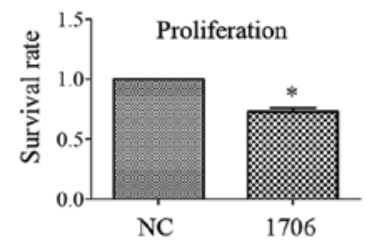

Figure 4. siRNA-mediated PARP1 inhibition suppresses the EGFR signaling pathway in C4-2 and DU145 cells. (A) Non-specific siRNA was used as a negative control (NC), and 3 PARP1 siRNAs (siRNA-1706, -2003 and -2907) were used to knock down PARP1 expression. siRNA-1706 was the most efficient and further used in the following experiment. (B) Following PARP1 knockdown by PARP1 siRNA-1706, EGFR was decreased in C4-2 cells. (C) Following PARP1 knockdown by PARP1 siRNA-1706 in C4-2 cells, docetaxel was used to induce apoptosis and expression of cleaved-caspase3 was detected via western blotting. (D) Compared with the NC group, EGFR expression in the PARP1 siRNA-1706 group was significantly decreased in Du145 cells. (E) Similar to the C4-2 cells, c-caspase 3 expression in the siRNA-1706 group was significantly increased by docetaxel treatment in Du145 cells. (F) Following PARP1 knockdown by PARP1 siRNA-1706 in Du145 cells, its proliferation was decreased. $n=3$. ${ }^{*} \mathrm{P}<0.05$ with respect to the negative control group. C-caspase3, cleaved-caspase3; C, control group; NC, negative control group; 1706, siRNA-1706 group; 2003, siRNA-2003 group; 2907, siRNA-2907 group.

signaling pathway. Notably, this function of PARP1-siRNA in PCa cells may be different from PARPi and may provide a potential therapy method for human CRPC.

PARP1 is an enzyme that catalyzes the covalent attachment of polymers of ADP-ribose (PAR) moieties on itself and its target proteins. PARP1 is overexpressed in various tumors and its overexpression is associated with poor overall survival (12-15). In fact, targeting DNA repair defects with PARP1 inhibitors (PARPi) is emerging as a cancer treatment strategy, especially in BRCA1 mutation cases. However, another suitable predictive biomarker is still required, since resistance to PARPi frequently occurs (16-18). For this reason, we chose PARP1-siRNA instead of PARPi in our study. We first investigated the effect of PARP1-siRNA on the progression and invasion capacity of PCa cells. The results revealed that PARP1-siRNA could act as a tumor progression and invasion suppressor in PCa cells, suppressing PCa cell proliferation, migration and invasion capacities in vitro and tumor growth in vivo.

Initiation of EMT is closely related to drug resistance, tumor relapse as well as metastasis (19). The expression changes of associated markers, such as E-cadherin and vimentin, are important characteristics of EMT (20). Impaired PARP-1 function promotes prostate tumorigenesis in vivo via TGF- $\beta$-induced EMT (21). However, our results revealed that PARP1-siRNA significantly altered EMT markers in PC3 cells in vitro and in vivo, which was visualized by the upregulation of E-cadherin and the downregulation of vimentin in PC3 cells with the knockdown of PARP1. These results indicated that PARP1-siRNA may suppress the progression and metastasis capacities of prostate cancer cells by suppressing EMT.

EGFR expression was required for primary and secondary sphere formation of prostate cancer cells (22), and EGFR is a key inducer of EMT in cancer progression $(23,24)$, since E-cadherin is the downstream of the EGFR signal (25). EGF binding to EGFR displays intrinsic tyrosine kinase activity and activates downstream signals via phosphorylation of key proteins. Among them, Akt/GSK-3 $\beta$ signaling is a key downstream target of EGFR signaling (26). In addition, Akt/GSK-3 $\beta$ signaling is crucial for the EMT of prostate cancer cells (27). PARPi inactivated GSK-3 $\beta$, which in turn enhanced PARPi-mediated PD-L1 upregulation (28). Thus, whether PARP1-siRNA could upregulate PD-L1 the same way as PARPi warrants further investigation. Our previous 
study revealed that PARP1-siRNA enhanced the activity of docetaxel against PC3 cells, and was associated with an accelerated suppression of the EGF/Akt/FOXO1 signaling pathway and was contrary to ABT88 (a PARPi) (10). However, GSK-3 $\beta$ is also downstream of the Akt signaling pathway. Therefore, we investigated whether PARP1-siRNA altered the phosphorylation status of GSK-3 $\beta$ in PC 3 cells. Our results revealed that PARP1-siRNA could clearly inhibit the expression of EGFR and p-GSK-3 $\beta$. Notably, whether overexpression of EGFR signaling could abolish the function of PARP1-siRNA warrants further investigation. Taking our previous and present study into consideration, PARP1siRNA, unlike ABT88 (10), may suppress EGF/EGFR/Akt signaling, with GSK-3 $\beta$ and FoxO1 as down targets, and is highly correlated with tumor cell growth, EMT and other properties.

Acquired cisplatin-resistant ovarian cancer cells expressed high levels of PARP-1 proteins, and silencing of PARP-1 increased cisplatin sensitivity in resistant cells (29). Notably, whether PARP1-siRNA can increase cisplatin sensitivity in resistant PCa cells warrants further investigation. Besides DNA damage repair function (30), emerging evidence also suggests that PARP1 has close connections with the transcriptional activities of the androgen receptor (AR) in PCa. Whether the function of PARP1-siRNA could regulate AR in PCa cells warrants further investigation.

In conclusion, the present study indicated that PARP1siRNA suppressed the growth, migration and invasion capacity of PCa cells. Our study also identified a novel role for PARP1siRNA in modulating EMT and EGFR/Akt/GSK-3 $\beta$ signaling in PCa cells. Furthermore, PARP1-siRNA may provide a potential new therapeutic approach for the treatment of $\mathrm{PCa}$, and we speculate that the signaling pathway associated with PARP1 knockout may be different from PARPi in PCa cells.

\section{Acknowledgements}

The present study was sustained in part by the National Natural Science Foundation of China (contract nos. 81402430 and 81602541).

\section{Competing interests}

The authors declare that they have no competing interests.

\section{References}

1. Siegel RL, Miller KD and Jemal A: Cancer Statistics, 2017. CA Cancer J Clin 67: 7-30, 2017.

2. Blessing AM, Rajapakshe K, Reddy Bollu L, Shi Y, White MA, Pham AH, Lin C, Jonsson P, Cortes CJ, Cheung E, et al: Transcriptional regulation of core autophagy and lysosomal genes by the androgen receptor promotes prostate cancer progression. Autophagy 13: 506-521, 2017.

3. Lu X, Horner JW, Paul E, Shang X, Troncoso P, Deng P, Jiang S, Chang Q, Spring DJ, Sharma P, et al: Erratum: Effective combinatorial immunotherapy for castration-resistant prostate cancer. Nature 543: 116, 2017.

4. Lin KY and Kraus WL: PARP Inhibitors for cancer therapy. Cell 169: 183, 2017.

5. Ray Chaudhuri A and Nussenzweig A: The multifaceted roles of PARP1 in DNA repair and chromatin remodelling. Nat Rev Mol Cell Biol 18: 610-621, 2017.
6. Schiewer MJ and Knudsen KE: Transcriptional roles of PARP1 in cancer. Mol Cancer Res 12: 1069-1080, 2014.

7. Asim M, Tarish F, Zecchini HI, Sanjiv K, Gelali E, Massie CE, Baridi A, Warren AY, Zhao W, Ogris C, et al: Synthetic lethality between androgen receptor signalling and the PARP pathway in prostate cancer. Nat Commun 8: 374, 2017.

8. Ning J, Wakimoto H, Peters C, Martuza RL and Rabkin SD: Rad51 degradation: Role in oncolytic virus-poly(ADP-ribose) polymerase inhibitor combination therapy in glioblastoma. J Natl Cancer Inst 109: 1-13, 2017.

9. Wu W, Zhu H, Liang Y, Kong Z, Duan X, Li S, Zhao Z, Yang D and Zeng G: Expression of PARP-1 and its active polymer PAR in prostate cancer and benign prostatic hyperplasia in Chinese patients. Int Urol Nephrol 46: 1345-1349, 2014.

10. Wu W, Kong Z, Duan X, Zhu H, Li S, Zeng S, Liang Y, Iliakis G, Gui $Z$ and Yang D: Inhibition of PARP1 by small interfering RNA enhances docetaxel activity against human prostate cancer PC3 cells. Biochem Biophys Res Commun 442: 127-132, 2013.

11. Lee JY and Kong G: Roles and epigenetic regulation of epithelialmesenchymal transition and its transcription factors in cancer initiation and progression. Cell Mol Life Sci 73: 4643-4660, 2016.

12. Rojo F, Garcia-Parra J, Zazo S, Tusquets I, Ferrer-Lozano J, Menendez S, Eroles P, Chamizo C, Servitja S, RamírezMerino N, et al: Nuclear PARP-1 protein overexpression is associated with poor overall survival in early breast cancer. Ann Oncol 23: 1156-1164, 2012.

13. Donizy P, Pietrzyk G, Halon A, Kozyra C, Gansukh T, Lage H, Surowiak P and Matkowski R: Nuclear-cytoplasmic PARP-1 expression as an unfavorable prognostic marker in lymph nodenegative early breast cancer: 15-year follow-up. Oncol Rep 31: 1777-1787, 2014.

14. Liu Y, Zhang Y, Zhao Y, Gao D, Xing J and Liu H: High PARP-1 expression is associated with tumor invasion and poor prognosis in gastric cancer. Oncol Lett 12: 3825-3835, 2016.

15. Galia A, Calogero AE, Condorelli R, Fraggetta F, La Corte A, Ridolfo F, Bosco P, Castiglione R and Salemi M: PARP-1 protein expression in glioblastoma multiforme. Eur J Histochem 56: e9, 2012.

16. Kurfurstova D, Bartkova J, Vrtel R, Mickova A, Burdova A, Majera D, Mistrik M, Kral M, Santer FR, Bouchal J and Bartek J: DNA damage signalling barrier, oxidative stress and treatmentrelevant DNA repair factor alterations during progression of human prostate cancer. Mol Oncol 10: 879-894, 2016.

17. Choi YE, Meghani K, Brault ME, Leclerc L, He YJ, Day TA, Elias KM, Drapkin R, Weinstock DM, Dao F, et al: Platinum and PARP inhibitor resistance due to overexpression of MicroRNA-622 in BRCA1-Mutant ovarian cancer. Cell Rep 14: 429-439, 2016.

18. Wang Y, Krais JJ, Bernhardy AJ, Nicolas E, Cai KQ, Harrell MI, Kim HH, George E, Swisher EM, Simpkins F and Johnson N: RING domain-deficient BRCA1 promotes PARP inhibitor and platinum resistance. J Clin Invest 126: 3145-3157, 2016.

19. Tong D, Liu Q, Liu G, Xu J, Lan W, Jiang Y, Xiao H, Zhang D and Jiang J: Metformin inhibits castration-induced EMT in prostate cancer by repressing COX2/PGE2/STAT3 axis. Cancer Lett 389: 23-32, 2017

20. Chaudhry P, Fabi F, Singh M, Parent S, Leblanc V and Asselin E: Prostate apoptosis response- 4 mediates TGF- $\beta$-induced epithelialto-mesenchymal transition. Cell Death Dis 5: e1044, 2014.

21. $\mathrm{Pu} \mathrm{H}$, Horbinski $\mathrm{C}$, Hensley PJ, Matuszak EA, Atkinson T and Kyprianou N: PARP-1 regulates epithelial-mesenchymal transition (EMT) in prostate tumorigenesis. Carcinogenesis 35: 2592-2601, 2014.

22. Day KC, Lorenzatti Hiles GL, Kozminsky M, Dawsey SJ, Paul A, Broses LJ, Shah R, Kunja LP, Hall C, Palanisamy N, et al: HER2 and EGFR overexpression support metastatic progression of prostate cancer to bone. Cancer Res 77: 74-85, 2017.

23. Liu XL, Zhang XT, Meng J, Zhang HF, Zhao Y, Li C, Sun Y, Mei QB, Zhang $F$ and Zhang T: ING5 knockdown enhances migration and invasion of lung cancer cells by inducing EMT via EGFR/PI3K/Akt and IL-6/STAT3 signaling pathways. Oncotarget 8: 54265-54276, 2017.

24. El Bezawy R, Cominetti D, Fenderico N, Zuco V, Beretta GL, Dugo M, Arrighetti N, Stucchi C, Rancati T, Valdagni R, et al: miR-875-5p counteracts epithelial-to-mesenchymal transition and enhances radiation response in prostate cancer through repression of the EGFR-ZEB1 axis. Cancer Lett 395: 53-62, 2017. 
25. Kim GT, Lee SH and Kim YM: Torilis japonica extract, a new potential EMT suppressor agent by regulation of EGFR signaling pathways. Int J Oncol 45: 1673-1679, 2014.

26. Wei A, Fan B, Zhao Y, Zhang H, Wang L, Yu X, Yuan Q, Yang D and Wang S: ST6Gal-I overexpression facilitates prostate cancer progression via the PI3K/Akt/GSK-3 $\beta / \beta$-catenin signaling pathway. Oncotarget 7: 65374-65388, 2016.

27. Guo H, Luo H, Yuan H, Xia Y, Shu P, Huang X, Lu Y, Liu X, Keller ET, Sun D, et al: Litchi seed extracts diminish prostate cancer progression via induction of apoptosis and attenuation of EMT through Akt/GSK-3 $\beta$ signaling. Sci Rep 7: 41656, 2017.
28. Jiao S, Xia W, Yamaguchi H, Wei Y, Chen MK, Hsu JM, Hsu JL, Yu WH, Du Y, Lee HH, et al: PARP inhibitor upregulates PD-L1 expression and enhances cancer-associated immunosuppression. Clin Cancer Res 23: 3711-3720, 2017.

29. Wang J, Kho DH, Zhou JY, Davis RJ and Wu GS: MKP-1 suppresses PARP-1 degradation to mediate cisplatin resistance. Oncogene 36: 5939-5947, 2017.

30. Schiewer MJ, Goodwin JF, Han S, Brenner JC, Augello MA, Dean JL, Liu F, Planck JL, Ravindranathan P, Chinnaiyan AM, et al: Dual roles of PARP-1 promote cancer growth and progression. Cancer Discov 2: 1134-1149, 2012. 